\title{
Layered Graphene Oxide Films for Enhanced Nonlinear Optics in Integrated Waveguides
}

\author{
Jiayang $\mathrm{Wu}^{1}$, Yunyi Yang ${ }^{1}$, Xingyuan $\mathrm{Xu}^{1}$, Yang $\mathrm{Qu}^{1}$, Linnan Jia ${ }^{1}$, Yuning Zhang ${ }^{1}$, Yao Liang ${ }^{1}$, \\ Sai T. Chu ${ }^{2}$, Brent E. Little ${ }^{3}$, Roberto Morandotti ${ }^{4}$, Baohua Jia ${ }^{1}$, and David Moss ${ }^{1, *}$ \\ ${ }^{I}$ Centre for Micro-Photonics, Swinburne University of Technology, Hawthorn, VIC 3122, Australia \\ ${ }^{2}$ City University of Hong Kong, Tat Chee Avenue, Hong Kong, China \\ ${ }^{3} X i$ 'an Institute of Optics and Precision Mechanics Precision Mechanics of CAS, Xi'an, China \\ ${ }^{4} I N R S$-Énergie, Matériaux et Télécommunications, 1650 Boulevard Lionel-Boulet, Varennes, Québec, Canada
}

\begin{abstract}
Enhanced Kerr optical nonlinearities of waveguides integrated with layered graphene oxide (GO) films is experimentally demonstrated. Up to $\sim 9.5-\mathrm{dB}$ enhancement of four-wave mixing conversion efficiency is achieved for a waveguide with 2 layers of GO.
\end{abstract}

Nonlinear photonic chips provide a powerful solution to generate and process signals all-optically with the added benefits of compact footprint, high stability, mass-producibility, and the potential to significantly reduce cost [1]. Although silicon has been the leading platform for integrated photonic devices, its high two-photon absorption (TPA) at near-infrared wavelengths poses a fundamental limitation to the nonlinear performance of silicon photonic devices in the telecommunications band [2-6]. Benefitting from the extraordinarily low losses - both linear and nonlinear high-index doped silica glass has been a successful integrated platform for nonlinear photonic devices [1,7-14]. Nevertheless, the relatively low Kerr nonlinearity as compared with silicon and silicon nitride limits the performance of nonlinear photonic devices made from this platform. Owing to its ease of preparation as well as its tunable material properties, graphene oxide (GO) has become a rising star of the graphene family [15-17]. Previously, we reported a giant Kerr nonlinear response of GO films of $4 \sim 5$ orders of magnitude higher than that of high-index doped silica glass [17]. Moreover, as compared with graphene, GO has much lower loss, a very large bandgap for greatly reduced TPA in the telecommunications band, and better capability for large-scale fabrication.
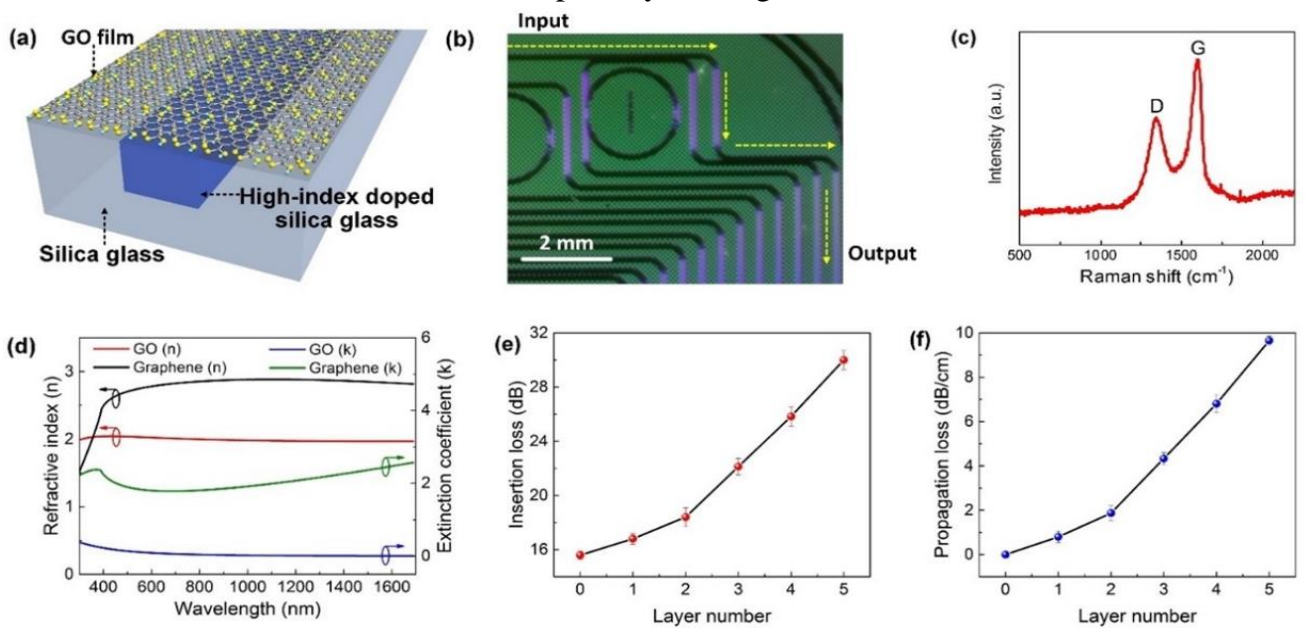

Fig. 1. (a) Schematic illustration of integrated waveguides coated with GO film. (b) Image of the hybrid integrated waveguide with two layers of GO. (c) Raman spectra of GO on the integrated chip. (d) Measured refractive indices and extinction coefficients of GO and graphene. (e) Measured insertion loss of a 1.5 -cm-long integrated waveguide with different numbers of GO layers. (f) Additional propagation loss of the integrated waveguide with different numbers of GO layers.

Here, we experimentally demonstrate an enhanced Kerr optical nonlinearity of GO coated integrated waveguides [17]. By designing and fabricating integrated waveguides with strong mode overlap with layered GO films, we achieve significantly enhanced four-wave mixing (FWM) efficiency in the GO hybrid integrated waveguides. A maximum conversion efficiency (CE) enhancement of $\sim 9.5 \mathrm{~dB}$ is experimentally achieved for a 1.5 -cm-long waveguide with 2 layers of GO. FWM measurements are also performed for different pump powers, wavelength detuning, GO coating lengths, and GO layer numbers. These results demonstrate the promise of layered GO films for enhanced nonlinear optics in integrated waveguides.

Figure 1(a) shows the GO-coated integrated waveguides made from high-index doped silica glass. The integrated waveguide is surrounded by silica except that the upper cladding was removed to enable coating the waveguide with GO films. The GO films, with a thickness of about $2 \mathrm{~nm}$ per layer, were introduced on top of the integrated waveguides via a solution-based transfer-free method with layer-by-layer deposition of GO films. The waveguides were fabricated 
via CMOS compatible processes $[9,10]$. An image of the integrated waveguides incorporating 2 layers of GO (Fig. 1(b)) is confirmed by Raman spectroscopic measurements (Fig. 1(c)). Figure 1(d) shows the in-plane refractive index $(n)$ and extinction coefficient $(k)$ of the GO film measured by spectral ellipsometry. For comparison, the refractive index and extinction coefficient of graphene are also shown. Figure 1(e) depicts the total insertion loss of the integrated waveguides with different numbers of GO layers. We employed a single-mode fibre (SMF) array for butt coupling. The coated GO layers only affected the propagation (not coupling) loss, which is shown in Fig. 1(f) vs of layers.

Figure 2 shows the FWM experimental results. The FWM spectra of a 1.5-cm-long integrated waveguide without GO and with 2 layers of GO are shown in Fig. 2(a). It can be seen that although the hybrid integrated waveguide had additional propagation loss $(2.6 \mathrm{~dB})$, it clearly shows enhanced idler output powers as compared with the same waveguide without GO. There is a maximum CE enhancement of $9.5 \mathrm{~dB}$, corresponding to a net $\mathrm{CE}$ enhancement of $6.9 \mathrm{~dB}$. For the integrated waveguide coated with 1 to 5 layers of GO, zoom-in spectra of the generated idlers for the same pump power coupled to the waveguide $(22 \mathrm{dBm})$ are shown in Fig. $2(\mathrm{~b})$. The output powers of the idler for various pump powers coupled to the waveguide without GO and with 2 layers of GO are shown in Fig. 2(c). The net CE enhancement for various pump powers coupled to the waveguide with 1 to 5 layers of GO is shown in Fig. 2(d). The variation in idler power when the pump wavelength was fixed at $1550 \mathrm{~nm}$ and the signal wavelength detuned around $1550 \mathrm{~nm}$ is shown in Fig. 2(e). The output powers for the idler light for a 1.5-cm-long integrated waveguide coated with different lengths of GO are depicted in Fig. 2(f).
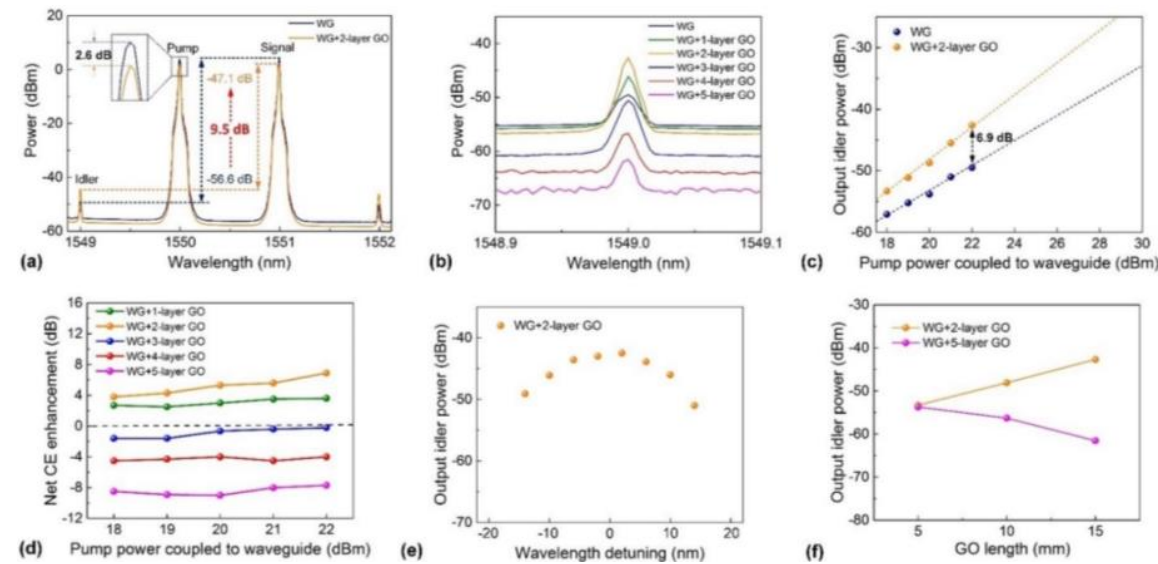

Fig. 2. (a) FWM spectra of the waveguide without GO and with 2 layers of GO. (b) Zoom in spectra of the idlers after FWM in the wav eguide with $0-5$ layers of GO. (c) Output powers of idler for various pump powers. (d) Net CE enhancements for various pump powers. (e) Power variations of the output idler when the signal wavelength was detuned. (f) Output powers of idler for the waveguide with different coating lengths of GO. The GO coating length in (a) $-(\mathrm{e})$ is $\sim 1.5 \mathrm{~cm}$. The pump power coupled to the waveguide in (a), (b), (e), (f) is $\sim 22 \mathrm{dBm}$. WG: waveguide.

\section{References}

[1] D. Moss et.al., "New CMOS-compatible platforms based on silicon nitride and Hydex for nonlinear optics," Nat. Photon., 7(8), 597-607, 2013.

[2] D. J. Moss, H. M. van Driel, and J. E. Sipe, "Dispersion in the anisotropy of optical third-harmonic generation in silicon," Opt. Lett., vol. 14, no. 1 , pp. $57-59,1989$.

[3] B. Corcoran, et al., "Green light emission in silicon through slow-light enhanced third-harmonic generation in photonic-crystal waveguides," Nature Photonics, vol. 3, no. 4, pp. 206-210, 2009. doi:10.1038/nphoton.2009.28.

[4] D. J. Moss, H. M. van Driel, and J. E. Sipe, "Third harmonic generation as a structural diagnostic of ion implanted amorphous and crystalline silicon," Appl. Phy. Lett., vol. 48, no. 17, pp. 1150, 1986.

[5] D. J. Moss, et al., "Ultrafast all-optical modulation via two-photon absorption in silicon-insulator waveguides," Electronics Letters, vol. 41, no. 6, pp. 320-321, 2005. DOI:10.1049/el:20058051

[6] M. R. E. Lamont et al.,"Two-photon absorption effects on self-phase-modulation-based 2R optical regeneration," Photonics Technology Letters, vol. 18, no. 10, pp. 1185-1187, 2006. DOI:10.1109/LPT.2006.874718.

[7] M. Ferrera et al., "All-optical 1st and 2nd order integration on a chip,” Optics Express, vol. 19, no. 23, pp. 23153-23161, 2011.

[8] M. Ferrera et al., "Low-power continuous-wave nonlinear optics in doped silica glass integrated waveguide structures," Nature Photonics, vol. 2, no. 12, pp. 737-740, 2008.

[9] J. Wu et.al., "Advanced photonic filters based on cascaded Sagnac loop reflector resonators in silicon-on-insulator nanowires," APL Photonics, 3(4), 046102, 2018.

[10] J. Wu et.al., "RF photonics: an optical microcombs' perspective," J. Sel. Top. Quantum Electron., 24(4), 1-20, 2018.

[11]X. Xu et.al., "Advanced RF and microwave functions based on an optical frequency comb source," Opt. Exp., 26(3), 2569-2583, 2018.

[12]X. Xu et al., "Broadband RF channelizer with an integrated frequency Kerr comb source," J. Lightw. Technol., 36(19), 4519-4526, 2018.

[13]X. Xu et.al., "Orthogonally polarized RF optical single sideband generation and dual-channel equalization based on an integrated micro-ring resonator," J. Lightw. Technol., 36(20), 4808-4818, 2018.

[14]X. Xu et.al., "Reconfigurable broadband microwave photonic intensity differentiator based on an integrated optical frequency comb source," APL Photonics, 2(9), 096104, 2017.

[15]K. Loh et.al., "Graphene oxide as a chemically tunable platform for optical applications," Nat. Chem., 2(12), 1015-1024, 2010.

[16]X. Zheng et.al., "In situ third-order non-linear response during laser reduction of GO thin films," Adv. Mater., 26(17), 2699-2703, 2014.

[17] Y. Yang et.al., "Enhanced four-wave mixing in waveguides integrated with graphene oxide," APL Photonics, 3(12), $120803,2018$. 